\title{
Supergravity and Cosmology
}

\section{Renata Kallosh*}

Stanford Institute for Theoretical Physics and Department of Physics, Stanford University, Stanford, CA 94305, USA

E-mail: kalloshestanford.edu

For cosmology we need General Relativity. Supergravity is the next natural step after General Relativity. Superstring theory is believed to be the most fundamental theory we know. However, string theory has an emergent concept of space-time. To use it in the context of the 4dimensional General Relativity and cosmology requires many intermediate steps. If in these steps some amount of supersymmetry, maximal or minimal or intermediate, is preserved, one finds consequences for cosmology, potentially supportable or falsifiable by observations.

2nd World Summit: Exploring the Dark Side of the Universe 25-29 June, 2018 - EDSU2018

University of Antilles, Pointe-à-Pitre, Guadeloupe, France

${ }^{*}$ Speaker. 
Fundamental idea following the discovery of General Relativity is the idea of local supersymmetry. Einstein's dream of unifying electromagnetism and gravity was realized starting with extended $N=2$ supergravity. The model does so by adding two real gravitino to the photon and the graviton. The first breakthrough into finiteness of quantum supergravity occurred via this unification: an explicit calculation of photon-photon scattering which was known to be divergent in the coupled bosonic Maxwell-Einstein system yielded a dramatic result : the new diagrams involving gravitinos cancelled the divergences found previously. Many more unexpected cancellation of the quantum loop divergences were discovered later in extended maximal supergravities with up to $\mathscr{N}=8$ supersymmetries.

However, supergravity models discovered in 1976 with linearly realized supersymmetry tend to have an easy descriptions of anti-de Sitter vacua with negative cosmological constant. The have difficulty in describing the Universe we see in observations, where we need to explain the origin of the positive cosmological constant, for example via a de Sitter vacua, as well as near de Sitter geometry describing a slow-roll potentials in inflationary cosmology.

Cosmology: from the sky to the fundamental physics

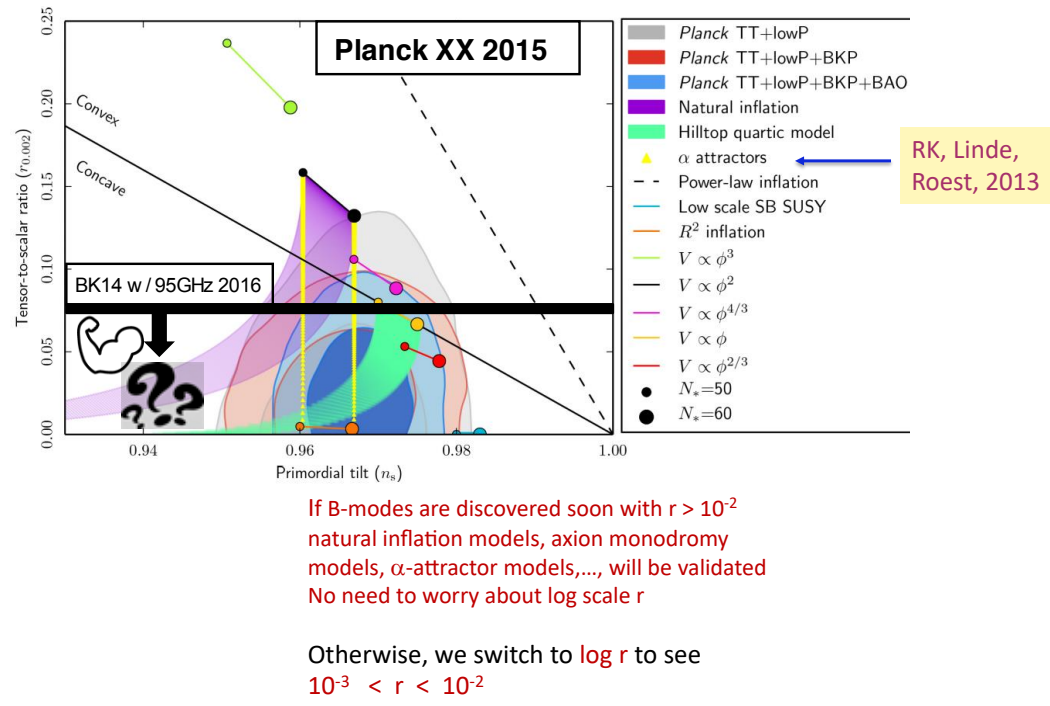

Figure 1: Planck $2015 n_{s}-r$ plane with blue area representing the $2 \sigma$ data.

In this talk we will describe the recently discovered De Sitter supergravity [1] with nilpotent superfield and based on it inflationary model building. We present inflationary models still consistent with Planck 2015 [2] in $n_{s}-r$ plane in Fig. 1. The models at present compatible with the data include the so-called $\alpha$-attractors [3] which we will describe in the context of supergravity. In fact, the most advanced geometric version of these models are using de Sitter supergravity with a nilpotent multiplet and a Kähler function [4]. We show the place of these models in Planck $2015 n_{s}-r$ plane in Fig. 1. We explain the geometric nature of these models in Fig. 2. The main feature of these models is a particular choice of the moduli space geometry, which is a Poincare disk. There is a corresponding Escher's picture where $R_{\text {Escher }}^{2}=3 \alpha$. 
We will explain the new M-theory/String theory inspired targets for B-modes beyond the well known satellite targets like $R^{2}$ and Higgs inflation, with $r$ of the order $3 \times 10^{-3}$. The new targets shown in Fig. 3 below a scan the region of $r$ between $10^{-2}$ and $10^{-3}$.

\section{De Sitter supergravity}

Standard linear $\mathscr{N}=1$ supersymmetry in four dimensions, as discovered by Gol'fand and Lichtman in 1971 and By Wess and Zumino in 1974 was the major paradigm in particle physics for decades. LHC was expected to produce super-partners of known particles, one Majorana fermion for each complex scalar and vice versa. So far we have not seen them, which of course is easy to explain if the super-partners are heavier than predicted. In any case, another issue with linear supersymmetry was realized in the context of cosmology. In all linearized supersymmetries with $\mathscr{N} \geq 1$ there are theorems, under certain conditions, supporting anti-de Sitter vacua with unbroken supersymmetry and forbidding de Sitter vacua with spontaneously broken supersymmetry.

Meanwhile, starting with 1998 discovery of the dark energy it become clear that de Sitter space offers a is a simple explanation of the Einstein equations with a positive cosmological constant. During the last two decades all data points towards the fact that a positive cosmological constant is still a possible explanation of the cosmological data. The difficulties (in absence of scalar fields) of standard linearized supersymmetry to produce such theories forced us to study a non-linear realization supersymmetry which was in fact discovered long time by Volkov and Akulov in 1972. This theory is equivalent to a theory with a constrained chiral multiplet where only a fermionic degree of freedom is present, and a scalar field is a bilinear of the fermion one

$$
S^{2}(x, \theta)=0 \quad \Rightarrow \quad S(x, \theta)=\frac{\psi \psi(x)}{2 F}+\sqrt{2} \theta \psi(x)+\theta^{2} F
$$

To explain a consistency between a positive cosmological constant and supersymmetry it was necessary to promote Volkov-Akulov theory, or a theory with the nilpotent multiplet, to a theory with a local supersymmetry. This was done in [1]. When such a multiplet is present in the action one finds that even in absence of scalar fields such supergravity actions have de Sitter vacua with spontaneously broken supersymmetry.

In 1996 J. Polchinski discovered in string theory the non-perturbative objects, called D-branes and a year later it was recognized that the theory of non-linearly realized supersymmetry of the Volkov-Akulov type is a property of the world-volume actions of all D-branes. Therefore it was also recognized that the KKLT uplifting mechanism in string theory is associated with a nilpotent multiplet, [5] and therefore also with de Sitter supergravity.

\section{2. $\alpha$-attractor models}

These models have an interesting property that the potentials for canonical fields are for Tmodels and E-models, respectively

$$
V^{T}=V_{0}\left(\tanh \frac{\phi}{\sqrt{6 \alpha}}\right)^{2 n}, \quad V^{E}=V_{0}\left(1-e^{\sqrt{\frac{2}{3 \alpha}} \phi}\right)^{2 n}
$$




\section{$\alpha$-attractors in supergravity}

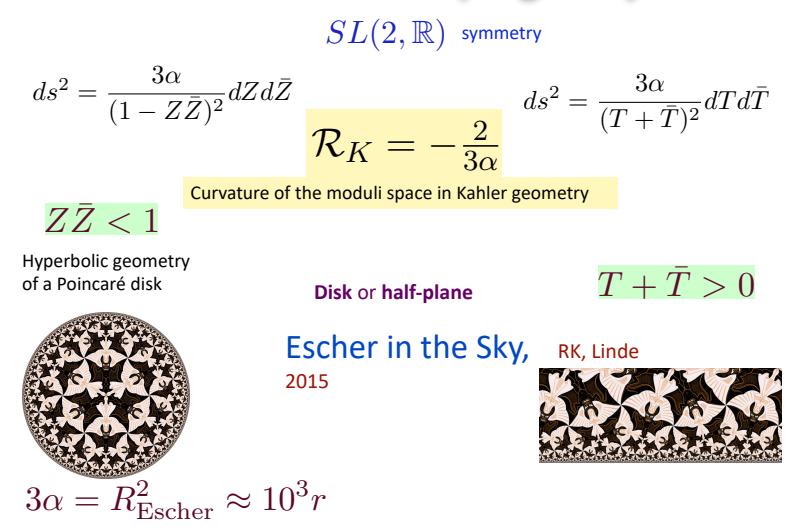

Figure 2: Planck $2015 n_{s}-r$ plane with blue area representing the $2 \sigma$ data.

At sufficiently small $\alpha$ these models have simple observables

$$
n_{s} \approx 1-\frac{2}{N}, \quad r \approx \alpha \frac{12}{N^{2}}
$$

Here $N$ is the number of e-foldings. In Fig. 1 there is a T-model with $n=1$, shown by two yellow lines for $N=50$ and $N=60$. All $\alpha$-attractor models are inside $2 \sigma$ for $n_{s}$ and waiting for the discovery of primordial gravitational waves, or new bounds on $r$ to identify the value of $\alpha$.

In Fig. 2 we explain some of the properties of these models in geometric terms. The T-models in geometric variables have a non-canonical kinetic term defined by the metric of a Poincaré disk and for example the $n=1$ potential is very simple, just $Z \bar{Z}$.

\section{New B-mode targets}

The region of $r$ between between $10^{-2}$ and $10^{-3}$ is expected to be a playground for the satellite B-mode missions. In the context of $\alpha$-attractor cosmological models the value of $\alpha$ can be arbitrary, as long as we only consider the embedding of the models in (2.1) into phenomenological $\mathscr{N}=1$ supergravity. The value of $\alpha=-\frac{2}{3 \mathscr{R}_{K}}$ related to the curvature of the Kähler manifold $\mathscr{R}_{K}$ as shown in Fig. 2 is not restricted, it can take any value. However, it was recently discovered in [6] that if one derives the corresponding kinetic terms starting from the maximal supersymmetry: M-theory in $\mathrm{d}=11$, superstring theory in $\mathrm{d}=10, \mathscr{N}=8$ supergravity in $\mathrm{d}=4$, the situation changes. Only integer values of $3 \alpha=1,2,3,4,5,6,7$ are possible. We have shown the corresponding values of $r$ according to (2.2) in the Fig. 3. These seven targets correspond to Escher's disks of $R_{\text {Escher }}^{2}=1,2,3,4,5,6,7$. 


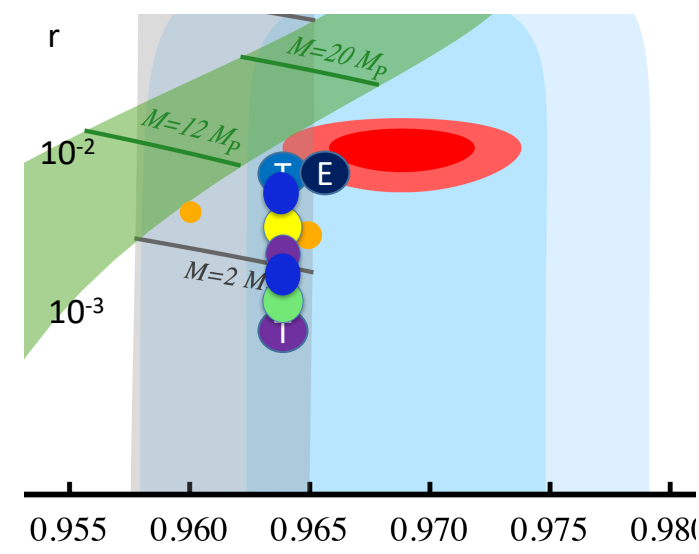

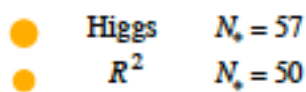

Seven new targets

Figure 3: The CMB-S4 figure represents the $n_{s}-r$ plane for the future data. The grey band shows one of the $\alpha$-attractor models. One can see the orange targets there for the Starobinsky and Higgs models. The seven new targets at various colors scan the region of $r$ between $10^{-2}$ and $10^{-3}$, which is expected to be probed by the satellite missions. These targets are derived from M-theory/String theory.

\section{References}

[1] E. A. Bergshoeff, D. Z. Freedman, R. Kallosh and A. Van Proeyen, Phys. Rev. D 92, no. 8, 085040 (2015) Erratum: [Phys. Rev. D 93, no. 6, 069901 (2016)] doi:10.1103/PhysRevD.93.069901, 10.1103/PhysRevD.92.085040 [arXiv:1507.08264 [hep-th]]. F. Hasegawa and Y. Yamada, "Component action of nilpotent multiplet coupled to matter in 4 dimensional $\mathscr{N}=1$ supergravity," JHEP 1510, 106 (2015) doi:10.1007/JHEP10(2015)106 [arXiv:1507.08619 [hep-th]].

[2] P. A. R. Ade et al. [Planck Collaboration], "Planck 2015 results. XX. Constraints on inflation,” Astron. Astrophys. 594 (2016) A20 doi:10.1051/0004-6361/201525898 [arXiv:1502.02114 [astro-ph.CO]].

[3] R. Kallosh, A. Linde and D. Roest, "Superconformal Inflationary $\alpha$-Attractors,” JHEP 1311 (2013) 198 doi:10.1007/JHEP11(2013)198 [arXiv:1311.0472 [hep-th]]. M. Galante, R. Kallosh, A. Linde and D. Roest, "Unity of Cosmological Inflation Attractors," Phys. Rev. Lett. 114 (2015) no.14, 141302 doi:10.1103/PhysRevLett.114.141302 [arXiv:1412.3797 [hep-th]]. J. J. M. Carrasco, R. Kallosh, A. Linde and D. Roest, "Hyperbolic geometry of cosmological attractors," Phys. Rev. D 92, no. 4, 041301 (2015) doi:10.1103/PhysRevD.92.041301 [arXiv:1504.05557 [hep-th]].

[4] R. Kallosh, A. Linde, D. Roest and Y. Yamada, “ $\overline{D 3}$ induced geometric inflation,” JHEP 1707, 057 (2017) doi:10.1007/JHEP07(2017)057 [arXiv:1705.09247 [hep-th]].

[5] R. Kallosh and T. Wrase, "Emergence of Spontaneously Broken Supersymmetry on an Anti-D3-Brane in KKLT dS Vacua,” JHEP 1412, 117 (2014) doi:10.1007/JHEP12(2014)117 [arXiv:1411.1121

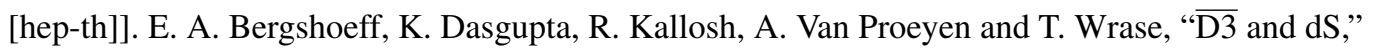
JHEP 1505, 058 (2015) doi:10.1007/JHEP05(2015)058 [arXiv:1502.07627 [hep-th]].

[6] S. Ferrara and R. Kallosh, "Seven-disk manifold, $\alpha$-attractors, and $B$ modes," Phys. Rev. D 94, no. 12, 126015 (2016) doi:10.1103/PhysRevD.94.126015 [arXiv:1610.04163 [hep-th]]. R. Kallosh, A. Linde, T. Wrase and Y. Yamada, "Maximal Supersymmetry and B-Mode Targets," JHEP 1704 (2017) 144 doi:10.1007/JHEP04(2017)144 [arXiv:1704.04829 [hep-th]]. 